\title{
n-COLOR PARTITIONS WITH WEIGHTED DIFFERENCES EQUAL TO MINUS TWO
}

\author{
A.K. AGARWAL \\ Mathematical Sciences Division \\ Institute of Advanced Study in \\ Science and Technology \\ Jawahar Nagar, Khanapara \\ Guwahati - 781022, INDIA
}

\section{R. BALASUBRANANIAN}

The Institute of Mathematical Sciences

C I T. Campus

Madras - 600113 , INDIA

(Received November 6, 1995)

\begin{abstract}
In this paper we study those $n$-color partitions of Agarwal and Andrews, 1987, in which each pair of parts has weighted difference equal to -2 Results obtained in this paper for these partitions include several combinatorial identities, recurrence relations, generating functions, relationships with the divisor function and computer produced tables. By using these partitions an explicit expression for the sum of the divisors of odd integers is given It is shown how these partitions arise in the study of conjugate and self-conjugate $n$-color partitions A combinatorial identity for self-conjugate $n$-color partitions is also obtained. We conclude by posing several open problems in the last section
\end{abstract}

KEY WORDS AND PHRASES: Partitions, combinatorial identities, recurrence relations, generating functions.

1991 AMS SUBJECT CLASSIFICATION CODES: Primary: 05A15, 05A17, 05A19, Secondary 11B37, $11 \mathrm{P} 81$

\section{INTRODUCTION, DEFINITIONS AND NOTATIONS}

$n$-color partitions (also called partitions with " $n$ copies of $n$ ) were introduced by Agarwal and Andrews in [2]. These are the partitions in which a part of size $n$, can come in $n$ different colors denoted by subscripts $n_{1}, n_{2}, \ldots, n_{n}$. Thus, for example, the $n$-color partitions of 3 are

$\begin{array}{lll}3_{1} & 3_{2} & 3_{3} \\ 2_{1} 1_{1} & 2_{2} 1_{1} & \\ 1_{1} 1_{1} 1_{1} & & \end{array}$

If $P(\nu)$ denote the number of $n$-color partitions of $\nu$, then it was shown in [2] that

$$
1+\sum_{\nu=1}^{\infty} P(\nu) q^{\nu}=\prod_{n=1}^{\infty}\left(1-q^{n}\right)^{-n}
$$

It was pointed out in [2] that since the right hand side of (1.1) is also a generating function for the MacMahon's plane partition function so the number of $n$-color partitions of $\nu$ equals the number of plane partitions of $\nu$ In terms of $n$-color partitions a class of new Rogers-Ramanujam type identities was given in the same paper. Further Rogers-Ramanujam Type identities using $n$-color partitions were found in [1] This was one of the advantages of studying $n$-color partitions as there are no Roers-Ramanujam Type identities for plane partitions In this paper we define conjugate and self-conjugate $n$-color partitions and obtain various combinatorial identities using these definitions. This will give another advantage of studying $n$-color partitions since there are no self-conjugate plane partitions. It is worthwhile to remark 
here that conjugate and self-conjugate $d(n)$-color partitions, where $d(n)$ is the numer of positive divisors of $n$ have been studied by Agarwal and Mullen in [3]

We now give some definitions which we shall use in this paper

DEFINITION 1. Let $\Pi=\left(a_{1}\right)_{b_{1}}+\left(a_{2}\right) b_{2}+\ldots+\left(a_{\tau}\right) b_{r}$ be an $n$-color partition of $\nu \quad$ We call $\left(a_{2}\right)_{a_{1}-b_{1}+1}$ the conjugate of $\left(a_{2}\right)_{b_{1}}$ An $n$-color partition of $\nu$ obtained from $\Pi$ by replacing each of its parts by its conjugate will be called the conjugate of $\Pi$ and will be denoted by $\Pi^{c}$ For example, if we consider $\Pi=5_{2}+3_{1}$, an $n$-color partition of 8 , then $\Pi^{c}=5_{5-2+1}+3_{3-1+1}=5_{4}+3_{3}$

DEFINITION 2. We shall call an $n$-color partition to be self-conjugate if it identical with its conjugate $\Pi^{c}$ Thus $5_{3}+3_{2}+1_{1}$ is a self-conjugate $n$-color partition of 9 .

DEFINITION 3 (see [2]). The weighted difference of any pair of parts $m_{\imath}, n$, is defined by $m-i-n-j$.

Throughout this paper $A(\nu)$ will denote the number of $n$-color partitions of positive integer $\nu$ where the weighted difference of each pair of parts is -2 Thus $A(\nu)=0$ if $\nu \leq 0 . A(m, \nu)$ will denote the number of partitions of $\nu$ enumerated by $A(\nu)$ with the added restriction that there be exactly $m$ parts Obviously, $A(m, \nu)=0$ for $\nu<m$

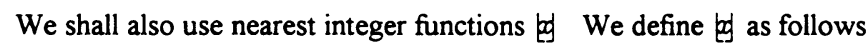

$$
\text { ed }=r \text {, }
$$

where $r$ is the unique integer such that

$$
x-\frac{1}{2} \leq r<x+\frac{1}{2} .
$$

\section{COMBINATORIAL IDENTITIES}

In this section we shall prove several combinatorial identities Our first identity is an easy consequence of the definition of conjugacy

THEOREM 2.1. Let $B(\nu)$ denote the number of $n$-color partitions of $\nu$ such that in each pair of parts $m_{\imath}, n_{\jmath}(m \geq n) n$ is the arithmetic mean of the subscripts $i$ and $j$. Then

$$
A(\nu)=B(\nu), \quad \forall \nu
$$

EXAMPLE. $A(5)=11$ The relevant partitions in this case are $5_{1}, 5_{2}, 5_{3}, 5_{4}, 5_{5}, 4_{4} 1_{1}, 3_{1} 2_{2}, 3_{2} 2_{1}$, $3_{3} 1_{1} 1_{1}, 2_{2} 1_{1} 1_{1} 1_{1}, 1_{1} 1_{1} 1_{1} 1_{1} 1_{1}$.

$B(5)$ is also equal to 11 , since in this case the relevant partitions are $5_{1}, 5_{2}, 5_{3}, 5_{4}, 5_{5}, 4_{1} 1_{1}, 3_{3} 2_{1}$, $3_{2} 2_{2}, 3_{1} 1_{1} 1_{1}, 2_{1} 1_{1} 1_{1} 1_{1}, 1_{1} 1_{1} 1_{1} 1_{1} 1_{1}$

PROOF. Conjugacy is the natural bijection between the two classes. To see this, let $\Pi$ be a partition enumerated by $A(\nu)$. That is, each pair of parts $m_{2}, n$, in $\Pi$ satisfies the condition

$$
m-n-i-j=-2 \text {. }
$$

We claim that each pair of parts $p_{q}, r_{s}$ in $\Pi^{c}$ satisfies the condition

We see that

$$
r=\frac{q+s}{2} \text {. }
$$

$$
\begin{array}{rlr}
p_{q}, r_{s} \in \Pi^{c} & \Rightarrow & p_{p-q+1}, r_{r-s+1} \in \Pi \\
& \Rightarrow p-(p-q+1)-r-(r-s+1)=-2, \\
& (\text { by (2.1)) }
\end{array}
$$

which is the same as (2.2).

To see the reverse implication let $\sigma$ be a partition enumerated by $B(\nu)$ That is, each pair of parts $p_{q}, r_{s} \in \sigma$ satisfies (2.2) We want to prove that each pair of parts $m_{\imath}, n_{\text {, }}$ in $\sigma^{c}$ satisfies (2 1)

Now 


$$
\begin{array}{lll}
m_{\imath}, n_{\jmath} \in \sigma^{c} & \Rightarrow \quad \begin{array}{l}
m_{m-\imath+1}, n_{n-\jmath+1} \in \sigma \\
(m-i+1)+(n-\jmath+1)
\end{array}=n
\end{array}
$$$$
\text { (by }\left(\begin{array}{ll}
2 & 2
\end{array}\right) \text { ), }
$$

\begin{tabular}{|c|c|c|}
\hline $\begin{array}{c}\text { Partitions enumerated } \\
\text { by } A(6)\end{array}$ & Conjugacy & $\begin{array}{c}\text { Partitions enumerated } \\
\text { by } B(6)\end{array}$ \\
\hline $6_{1}$ & $\longleftrightarrow$ & $\overline{6_{6}}$ \\
\hline $6_{2}$ & $\longleftrightarrow$ & $6_{5}$ \\
\hline $6_{3}$ & $\longleftrightarrow$ & $6_{4}$ \\
\hline $6_{4}$ & $\longleftrightarrow$ & $6_{3}$ \\
\hline $6_{5}$ & $\leftrightarrow$ & $6_{2}$ \\
\hline $6_{6}$ & $\longleftrightarrow$ & $6_{1}$ \\
\hline $5_{5} 1_{1}$ & $\longleftrightarrow$ & $5_{1} 1_{1}$ \\
\hline $4_{2} 2_{2}$ & $\longleftrightarrow$ & $4_{3} 2_{1}$ \\
\hline $4_{3} 2_{1}$ & $\longleftrightarrow$ & $4_{2} 2_{2}$ \\
\hline $4_{4} 1_{1} 1_{1}$ & $\longleftrightarrow$ & $4_{1} 1_{1} 1_{1}$ \\
\hline $3_{1} 3_{1}$ & $\longmapsto$ & $3_{3} 3_{3}$ \\
\hline $3_{3} 1_{1} 1_{1} 1_{1}$ & $\longleftrightarrow$ & $3_{1} 1_{1} 1_{1} 1_{1}$ \\
\hline $2_{1} 2_{1} 2_{1}$ & $\longmapsto$ & $2_{2} 2_{2} 2_{2}$ \\
\hline $2_{2} 1_{1} 1_{1} 1_{1} 1_{1}$ & $\longmapsto$ & $2{ }_{1} 1_{1} 1_{1} 1_{1} 1_{1}$ \\
\hline $1_{1} 1_{1} 1_{1} 1_{1} 1_{1} 1_{1}$ & $\longleftrightarrow$ & $1_{1} 1_{1} 1_{1} 1_{1} 1_{1} 1_{1}$ \\
\hline
\end{tabular}

To illustrate the bijection of Theorem 2.1, we provide the example for $\nu=6$

REMARK. Using the idea of conjugacy, partition identities have been obtained for ordinary partitions [4, Theorem 343, p. 274] and for $d(n)$-color partitions [3, Theorem 41, p 128] Following the method of proof of Theorem 21 , one can prove its following generalization

THEOREM 2.2. Let $A^{k}(\nu)$ denote the number of $n$-color partitions of $\nu$ such that each pair of parts has a weighted difference equal to $k-2$ Let $B^{k}(\nu)$ denote the number of $n$-color partitions of $\nu$ such that each pair of parts $m_{\imath}, n_{j}$ satisfies the condition $i+j-2 n=k$ Then $A^{k}(\nu)=B^{k}(\nu)$

REMARK. Theorem 2.1 is a particular case $k=0$ of Theorem 2.2 .

Theorem 21 is a combinatorial identity between two $n$-color partition functions Our next theorem is a combinatorial identity between an $n$-color partition function on one side and an ordinary partition function on the other side

THEOREM 2.3. Let $C(m, \nu)$ denote the number of ordinary partitions of all numbers $\leq \nu$ with minimum part $m$ and the differences between parts 0 or $m-1$ Then

$$
A(m, \nu)=C(m, \nu)
$$

EXAMPLE. Consider the case in which $\nu=11$ and $m=3$ We see that $A(3,11)=5$, since the relevant partitions are $9_{9} 1_{1} 1_{1}, 7_{6} 2_{1} 2_{1}, 6_{4} 3_{1} 2_{2}, 5_{3} 3_{1} 3_{1}, 4_{1} 4_{1} 3_{2}$; also $C(3,11)=5$; in this case the relevant partitions are: $3,3+3,3+5,3+3+3,3+3+5$.

PROOF. For $m=1$, the theorem is obviously true since $A(1, \nu)=\nu$, the relevant partitions are $\nu_{1}, \nu_{2}, \ldots, \nu_{\nu}$; also $C(1, \nu)=\nu$, in this case the relevant partitions are

$$
1,1+1,1+1+1, \ldots \underbrace{1+1+\ldots+1}_{\nu} .
$$

Now we consider the case when $m \geq 2$. In this case we shall first prove that

$$
A(m, \nu)=A(m, \nu-m)+A(m, \nu-2 m+1)-A(m, \nu-3 m+1)+1 \text {. }
$$


To prove (2 4), we split the partitions enumerated by $A(m, \nu)$ into three classes

(i) those that do not contain $k_{k}(k \geq 1)$ as a part,

(ii) those that contain $1_{1}$ as a part, and

(iii) those that contain $k_{k},(k>1)$ as a part but not $1_{1}$

We now transform the partitions in class (i) by deleting 1 from each part ignoring the subscripts Obviously, the transformed partition still satisfies the weighted difference condition so it will be a partition enumerated by $A(m, \nu-m)$. In class (ii) we observe that $1_{1}$ can appear only with $k_{k}(k \geq 1)$ in order to satisfy the weighted difference condition. Also, no two parts $k_{k}$ and $l_{l}$ with $k>1$ and $l>1$ can appear together This implies that corresponding to each value of $m(m>1)$, there is one and only one partition in class (ii) Finally, we transform the partitions in class (iii) by replacing $k_{k}$ by $(k+1)_{k-1}$ and then subtracting 2 from each part This will give a partition of $\nu-2 m+1$ into $m$ parts with $(k-1)_{k-1}$ as a part. Therefore, the actual number of partitions which belong to class (iii) is $A(m, \nu-2 m+1)-A(m, \nu-3 m+1)$, where $A(m, \nu-3 m+1)$ is the number of partitions of $\nu-2 m+1$ into $m$ parts which are free from the parts like $k_{k}(k \geq 1)$. The above transformations clearly prove (2 4)

Now we set

$$
D(m, \nu)=A(m, \nu)-A(m, \nu-m), \forall \nu
$$

Then

$$
D(m, \nu)=\left\{\begin{array}{l}
0 \text { if } \nu<m \\
D(m, \nu-2 m+1)+1, \text { if } \nu \geq m
\end{array} .\right.
$$

Next, we set

$$
E(m, \nu)=D(m, \nu)-D(m, \nu-2 m+1), \quad \forall \nu .
$$

Then

$$
E(m, \nu)= \begin{cases}0 & \text { if } \nu<m \\ 1 & \text { if } \nu \geq m\end{cases}
$$

Hence

$$
\begin{aligned}
\sum_{\nu=2}^{\infty} E(m, \nu) q^{\nu} & =0+\sum_{\nu=m}^{\infty} E(m, \nu) q^{\nu} \\
& =\sum_{\nu=m}^{\infty} q^{\nu} \\
& =\frac{q^{m}}{1-q}
\end{aligned}
$$

Using the extreme of the foregoing string of equations in (27) and then the resulting equation in (25), we get

$$
\begin{aligned}
\sum_{\nu=2}^{\infty} A(m, \nu) q^{\nu} & =\frac{q^{m}}{(1-q)\left(1-q^{m}\right)\left(1-q^{2 m-1}\right)} \\
& =\sum_{\nu=2}^{\infty} C(m, \nu) q^{\nu}
\end{aligned}
$$

comparing the coefficients of $q^{\nu}$, we get

$$
A(m, \nu)=C(m, \nu) \text { for } \quad 2 \leq m \leq \nu .
$$

This completes the proof of Theorem 23. 
NOTE. We remark here that the first part of (2.9) can, alternatively, be proved by using (24) Since if $f_{m}(q)=\sum_{\nu=2}^{\infty} A(m, \nu) q^{\nu}$ then (24) yields

$$
f_{m}(q)=q^{m} f_{m}(q)+q^{2 m-1} f_{m}(q)-q^{3 m-1} f_{m}(q)+q^{m}(1-q)^{-1} .
$$

\section{GENERATING FUNCTIONS}

Our discussion in the preceding section leads us to the following

THEOREM 3.1.

(i)

$$
\sum_{\nu=1}^{\infty} A(1, \nu) q^{\nu}=\frac{q}{(1-q)^{2}}
$$

(ii)

$$
\sum_{\nu=2}^{\infty} A(m, \nu) q^{\nu}=\frac{q^{m}}{(1-q)\left(1-q^{m}\right)\left(1-q^{2 m-1}\right)}, m \geq 2
$$

(iii)

$$
\sum_{\nu=1}^{\infty} A(\nu) q^{\nu}=\frac{q}{(1-q)^{2}}+\sum_{m=2}^{\infty} \frac{q^{m}}{(1-q)\left(1-q^{m}\right)\left(1-q^{2 m-1}\right)}
$$

\section{AN EXPLICIT FORMULA}

In this section we given an explicit formula for $A(m, \nu)$ We prove the following

THEOREM 4.1. Let $r$ be the quotient of $\frac{\nu}{m}$ and $k$ denotes the nearest integer function defined by (12) above Then

$$
A(m, \nu)=\left\{\begin{array}{l}
1 \text { if } m \geq \frac{\nu+1}{2} \\
\sum_{\jmath=0}^{r-1}\left\lfloor\frac{\nu-m_{\jmath}}{2 m-1}\right\rfloor, \quad \text { if } m<\frac{\nu+1}{2} .
\end{array}\right.
$$

PROOF. If $m \geq \frac{\nu+1}{2}$, then clearly $A(m, \nu)=1$ since the only relevant partition is

$$
(\nu-m+1)_{\nu-m+1} \underbrace{1_{1} 1_{1} \ldots 1_{1}}_{m-1 \text { parts }}
$$

so we consider the case when $m<\frac{\nu+1}{2}$. In (2.6) above we write

$$
\nu=k(2 m-1)+\ell \text {. }
$$

Then

$$
\begin{aligned}
D(m, k(2 m-1)+\ell) & =D(m,(k-1)(2 m-1)+\ell)+1 \\
& =D(m,(k-2)(2 m-1)+\ell)+2 \\
& \cdots \\
& =D(m, \ell)+k \\
& = \begin{cases}k+1 & \text { if } \ell \geq m \\
k & \text { if } \ell<m\end{cases}
\end{aligned}
$$

Hence

$$
D(m, \nu)=\left\lfloor\frac{\nu}{2 m-1}\right\rfloor .
$$

(2.5) in view of (4.1) can be written as

$$
A(m, \nu)=\left\{\begin{array}{l}
0 \text { if } \nu<m \\
A(m, \nu-m)+\left\lfloor\frac{\nu}{2 m-1}\right\rfloor \text { if } \nu \geq m
\end{array}\right.
$$

In (4.2) we write $\nu=r m+s$, then 


$$
\begin{aligned}
A(m, r m+s) & =a(m,(r-1) m+s)+\left\lfloor\frac{\nu}{2 m-1}\right\rfloor \\
& =A(m,(r-2) m+s)+\left\lfloor\frac{\nu-m}{2 m-1}\right\rfloor+\frac{\nu}{2 m-1} \\
& \cdots \\
& \left.=\left\lfloor\frac{\nu-(r-1) m}{2 m-1}\right\rfloor+\frac{\nu-(r-2) m}{2 m-1}\right\rfloor+\cdots+\left\lfloor\frac{\nu}{2 m-1}\right. \\
& =\sum_{\jmath=0}^{r-1}\left\lfloor\frac{\nu-m \jmath}{2 m-1}\right\rfloor
\end{aligned}
$$

This proves Theorem 41

\section{A RELATIONSHIP WITH DIVISOR FUNCTION}

In this section we shall prove a relationship between $A(\nu)$ and $d(\nu)$ where $d(\nu)$ denotes the number of positive divisors of $\nu$

THEOREM 5.1. Let a sequence $\left\{b_{\nu}\right\}$ be defined by $b_{1}=1, b_{\nu}=d(2 \nu-1)-d\left(\frac{\nu-1}{2}\right)-1, \nu \geq 2$ Let $\left\{B_{\nu}\right\}$ be the sequence of the partial sums of $b_{\nu}$ Then

$$
b_{\nu}=A(\nu)-2 A(\nu-1)+A(\nu-2), \nu \geq 1
$$

hence,

$$
B_{\nu}=A(\nu)-A(\nu-1), \quad \nu \geq 1
$$

PROOF. From Theorem 31 (iii), we have

$$
\begin{aligned}
(1-q)^{2} \sum A(\nu) q^{\nu} & =q+\sum_{m \geq 2} \frac{q^{m}(1-q)}{\left(1-q^{m}\right)\left(1-q^{2 m-1}\right)} \\
& =\sum_{m=1}^{\infty} \frac{q^{m}(1-q)}{\left(1-q^{m}\right)\left(1-q^{2 m-1}\right)}-\frac{q}{1-q}+q \\
& =\sum_{m=1}^{\infty} q^{m}\left\{\frac{\left(1+q^{m}\right)\left(1-q^{m}\right)-q\left(1-q^{2 m-1}\right)}{\left(1-q^{m}\right)\left(1-q^{2 m-1}\right)}\right\}-\frac{q^{2}}{(1-q)} \\
& =\sum_{m=1}^{\infty} q^{m}\left\{\frac{1+q^{m}}{1-q^{2 m-1}}-\frac{q}{\left(1-q^{m}\right)}\right\}-\frac{q^{2}}{(1-q)} \\
& =\sum_{m=1}^{\infty} \frac{q^{m}}{1-q^{2 m-1}}+\sum \frac{q^{2 m}}{1-q^{2 m-1}}-\sum \frac{q^{m+1}}{1-q^{m}}-\frac{q^{2}}{1-q} \\
& =a(q)+b(q)-c(q)-\frac{q^{2}}{1-q}, \text { say }
\end{aligned}
$$

Now writing the even $m$ 's and odd $m$ 's separately in $c(q)$, we get

$$
\begin{aligned}
& c(q)=\sum_{\ell \geq 1} \frac{q^{2 \ell+1}}{1-q^{2 \ell}}+\sum_{\ell \geq 1} \frac{q^{2 \ell}}{1-q^{2 \ell-1}} \\
& =\sum_{\ell \geq 1} \frac{q^{2 \ell+1}}{1-q^{2 \ell}}+b(q)=e(q)+b(q), \text { say } \\
& q^{-1} e(q)=\sum_{\ell \geq 1} \frac{q^{2 \ell}}{1-q^{2 \ell}} ; \text { Replacing } q \text { by } \sqrt{q}, \text { we get } \\
& q^{-\frac{1}{2}} e\left(q^{\frac{1}{2}}\right)=\sum_{\ell \geq 1} \frac{q^{\ell}}{1-q^{\ell}}=\sum d(n) q^{n}
\end{aligned}
$$


Hence

$$
\begin{aligned}
& e\left(q^{\frac{1}{2}}\right)=\sum d(n) q^{n+\frac{1}{2}} \\
& e(q)=\sum_{n \geq 1} d(n) q^{2 n+1}=\sum_{m \geq 1} d\left(\frac{m-1}{2}\right) q^{m} .
\end{aligned}
$$

Thus

$$
c(q)=\sum_{m \geq 1} d\left(\frac{m-1}{2}\right) q^{m}+b(q) .
$$

Now

$$
\begin{gathered}
a(q)=\sum_{m=1}^{\infty} \frac{q^{m}}{1-q^{2 m-1}}=\sum_{m=1}^{\infty} q^{m} \sum_{k \geq 1} q^{(2 m-1)(k-1)} \\
=\sum q^{2 m k-m-k+1}
\end{gathered}
$$

Hence

$$
\begin{gathered}
q^{-1} a\left(q^{2}\right)=\sum_{m, k} q^{4 m k-2 m-2 k+1}=\sum_{m \geq 1, k \geq 1} q^{(2 m-1)(2 k-1)} \\
=\sum_{n \text { odd }} d(n) q^{n}=\sum_{m \geq 1} d(2 m-1) q^{2 m-1} \\
a\left(q^{2}\right)=\sum d(2 m-1) q^{2 m} .
\end{gathered}
$$

Hence

$$
a(q)=\sum_{m \geq 1} d(2 m-1) q^{m}
$$

Putting these results together, we get,

$$
\begin{gathered}
(1-q)^{2} \sum_{\nu} A(\nu) q^{\nu}=\sum_{m \geq 1} d(2 m-1) q^{m}-\sum d\left(\frac{m-1}{2}\right) q^{m}-\frac{q^{2}}{(1-q)} \\
\sum_{m \geq 1}\left(d(2 m-1)-d\left(\frac{m-1}{2}\right)\right) q^{m}-\sum_{m \geq 2} q^{m}
\end{gathered}
$$

On comparing the coefficients of $q^{\nu}$ in the last equation, we are led to (5 1) $\quad$ (5 2) follows from (5 1) immediately once we note that the right-hand side of (5.2) is the nth partial sum of the right-hand side of (5.1) This completes the proof of Theorem 51.

REMARK. We remark here that from Theorem 5 1, we get an algorithm to compute the values of $A(\nu)$ The procedure is illustrated in the following table

\begin{tabular}{|r|r|r|r|}
\hline \multicolumn{1}{|c|}{$\nu$} & \multicolumn{1}{|c|}{$b_{\nu}$} & $B_{\nu}$ & $A(\nu)$ \\
\hline 1 & 1 & 1 & 1 \\
2 & 1 & 2 & 3 \\
3 & 0 & 2 & 5 \\
4 & 1 & 3 & 8 \\
5 & 0 & 3 & 11 \\
6 & 1 & 4 & 15 \\
7 & -1 & 3 & 18 \\
8 & 3 & 6 & 24 \\
9 & -2 & 4 & 28 \\
10 & 1 & 5 & 33 \\
11 & 1 & 6 & 39 \\
12 & 1 & 7 & 46 \\
\hline
\end{tabular}


From the table it is clear that each $A(\nu)$ equals the sum of the number immediately above it in the table and the number in the same row in the preceding column.

\section{6. n-COLOR PARTITIONS WITH WEIGHTED DIFFERENCES - 2 AND $k_{k}(k \geq 1)$ AS A PART.}

It is easily seen that $D(m, \nu)$ defined by (25) above is the number of those partitions which are enumerated by $A(m, \nu)$ and have $k_{k}(k \geq 1)$ as a part Let $D(\nu)$ equal $\sum_{m} D(m, \nu)$ Following the methods of preceding sections we can easily prove the following results:

THEOREM 6.1. Let $F(m, \nu)$ denote the number of ordinary partitions of all numers $\leq \nu$ into parts where the lowest part is $m$ which does not repeat and the differences between parts are 0 or $m-1$ Then

$$
D(m, \nu)=F(m, \nu) .
$$

EXAMPLE. Consider the case when $m=2$ and $\nu=11$. We see that $D(2,11)=4$, since the relevant partitions are $10_{10} 1_{1}, 9_{7} 2_{2}, 8_{4} 3_{3}, 7_{1} 4_{4}$, also, $F(2,11)=4$, since in this case the relevant partitions are

$$
2,2+3,2+3+3,2+3+3+3
$$

THEOREM 6.2.

(i)

$$
\sum_{\nu=1}^{\infty} D(1, \nu) q^{\nu}=\frac{q}{1-q}
$$

$$
\sum_{\nu=2}^{\infty} D(m ; \nu) q^{\nu}=\frac{q^{m}}{(1-q)\left(1-q^{2 m-1}\right)}
$$

(iii) $\quad \sum_{\nu=1}^{\infty} D(\nu) q^{\nu}=\frac{q}{(1-q)}+\sum_{m=2}^{\infty} \frac{q^{m}}{(1-q)\left(1-q^{2 m-1}\right)}$.

THEOREM 6.3.

$$
D(m, \nu)=\left\lfloor\frac{\nu}{2 m-1}\right\rfloor
$$

THEOREM 6.4. Let $k$ be the quotient of $\frac{\nu}{2 m-1}$ Then

$$
A(m, \nu)=\sum_{j=1}^{k-1} D(m, \nu-m j) .
$$

\section{THEOREM 6.5.}

$$
D(\nu)-D(\nu-1)=\left\{\begin{array}{ccc}
1 & \text { if } & \nu=1 \\
d(2 \nu-1)-1 & \text { if } & \nu>1
\end{array}\right.
$$

Using Theorems 6.3 and 6.5 , we shall now prove the following explicit expression for the sum of the divisors of odd numbers:

\section{THEOREM 6.6.}

$$
\sum_{j=1}^{\nu} d(2 j-1)=\sum_{m=1}^{\nu}\left\lfloor\frac{\nu}{2 m-1}\right\rfloor .
$$

PROOF. Replacing $\nu$ by $1,2, \ldots, \nu$ in Theorem 65 and then adding all equations, we get 


$$
D(\nu)=\sum_{j=1}^{\nu} d(2 j-1)-(\nu-1)
$$

that is

$$
1+\sum_{m \geq 2}^{\nu} D(m, \nu)=\sum_{j=1}^{\nu} d(2 j-1)-(\nu-1) .
$$

This proves Theorem 6.6 by Theorem 6.3

\section{A COMBINATORIAL IDENTITY FOR SELF-CONJUGATE $\boldsymbol{n}$-COLOR PARTITIONS}

In this section we shall prove a combinatorial identity which involves self-conjugate $n$-color partitions defined in Section 1 above.

THEOREM 7.1. Let $F(\nu)$ denote the number of self-conjugate $n$-color partitions of $\nu$ and $G(\nu)$ denote those $n$-color partitions of $\nu$ where each pair of parts has weighted difference greater than 1 and even parts appear with even subscripts and odd with odd Then $F(\nu)=G(\nu)$ for all $\nu$.

EXAMPLE. $F(7)=5$; the relevant partitions are

$$
\begin{aligned}
& 7_{4}, 5_{3}+1_{1}+1_{1}, 3_{2}+3_{2}+1_{1}, 3_{2}+1_{1}+1_{1}+1_{1}+1_{1}, 1_{1}+1_{1}+1_{1}+1_{1}+1_{1}+1_{1}+1_{1} \\
& G(7)=5, \text { in this case the relevant partitions are } 7_{1}, 7_{3}, 7_{5}, 7_{7}, 6_{2}+1_{1}
\end{aligned}
$$

PROOF. We observe that if an $n$-oder partition is self-conjugate then in each part $m_{\imath}$ of it, $m$ must be odd Because, $m_{\imath}=m_{m-\imath+1} \Rightarrow m=2 i-1$. Thus if we ignore the subscripts of all parts in a selfconjugate $n$-color partition of $\nu$, we get a unique ordinary partition of $\nu$ into odd parts. Conversely, if we consider an ordinary partition of $\nu$ into odd parts and replace each part $2 a-1$ by $(2 a-1)_{a}$ We get a unique self-conjugate $n$-color partition of $\nu$. This bijection shows that the number of self-conjugate $n$ color partitions of $\nu$ equals the number of ordinary partitions of $\nu$ into odd parts. That is,

$$
1+\sum_{\nu=1}^{\infty} F(\nu) q^{\nu}=\prod_{n=1}^{\infty} \frac{1}{1-q^{2 n-1}} .
$$

Now an appeal to the following theorem [1, Theorem 1.4, p. 301]

"Let $R(\nu)$ denote the number of $n$-color partitions of $\nu$ such that each pair of parts has weighted difference greater than 1 and even parts appear with even subscripts and odd with odd Let $S(\nu)$ denote the number of ordinary partitions of $\nu$ into distinct parts. Then

$$
R(\nu)=S(\nu) "
$$

proves Theorem 7.1.

\section{A TABLE FOR $A(m, \nu)$}

In this section we give a brief table of $A(m, \nu)$ which was obtained on computer by using the recurrence relation (2 4) above. The following observations can serve as a check on the table

$\begin{array}{lll}\text { Observation 1 } & A(1, \nu) & =\nu, \quad \forall \nu \\ \text { Observation 2 } & A(m, \nu) & =1, \text { if } m \geq \frac{\nu+1}{2} \\ \text { Observation 3 } & A(m, 2 m) & =2 \\ \text { Observation 4 } & A(m, 2 m+1) & =2\end{array}$

We remark here that Observation 1 was proved in the proof of Theorem 23 while Observation 2 in the proof of Theorem 4.1 Observations 3 and 4 follow immediately once we note that the only partitions enumerated by $A(m, 2 m)$ are

$$
(m+1)_{m+1} \underbrace{1_{1} 1_{1} \ldots 1_{1}}_{m-1} \text { and } \underbrace{2_{1} 2_{1} \ldots 2_{1}}_{m},
$$


and those enumerated by $A(m, 2 m+1)$ are

$$
(m+2)_{m+2} \underbrace{1_{1} 1_{1} \ldots 1_{1}}_{m-1} \text { and } 3_{2} \underbrace{2_{1} 2_{1} \ldots 2_{1}}_{m-1} .
$$

\begin{tabular}{|c|c|c|c|c|c|c|c|c|c|c|c|c|c|c|c|}
\hline$\nu / m$ & 1 & 2 & 3 & 4 & 5 & 6 & 7 & 8 & 9 & 10 & 11 & 12 & 13 & 14 & 15 \\
\hline 1 & 1 & & & & & & & & & & & & & & \\
\hline 2 & 2 & 1 & & & & & & & & & & & & & \\
\hline 3 & 3 & 1 & 1 & & & & & & & & & & & & \\
\hline 4 & 4 & 2 & 1 & 1 & & & & & & & & & & & \\
\hline 5 & 5 & 3 & 1 & 1 & 1 & & & & & & & & & & \\
\hline 6 & 6 & 4 & 2 & 1 & 1 & 1 & & & & & & & & & \\
\hline 7 & 7 & 5 & 2 & 1 & 1 & 1 & 1 & & & & & & & & \\
\hline 8 & 8 & 7 & 3 & 2 & 1 & 1 & 1 & 1 & & & & & & & \\
\hline$\overline{9}$ & 9 & 8 & 4 & 2 & 1 & 1 & 1 & 1 & 1 & & & & & & \\
\hline 10 & 10 & 10 & 4 & 2 & 2 & 1 & 1 & 1 & 1 & 1 & & & & & \\
\hline 11 & 11 & 12 & 5 & 3 & 2 & 1 & 1 & 1 & 1 & 1 & 1 & & & & \\
\hline 12 & 12 & 14 & 6 & 4 & 2 & 2 & 1 & 1 & 1 & 1 & 1 & 1 & & & \\
\hline 13 & 13 & 16 & 7 & 4 & 2 & 2 & 1 & 1 & 1 & 1 & 1 & 1 & 1 & & \\
\hline 14 & 14 & 19 & 8 & 4 & 3 & 2 & 2 & 1 & 1 & 1 & 1 & 1 & 1 & 1 & \\
\hline 15 & 15 & 21 & 9 & 5 & 4 & 2 & 2 & 1 & 1 & 1 & 1 & 1 & 1 & 1 & 1 \\
\hline
\end{tabular}

\section{CONCLUSION}

The most obvious questions arising from this work are:

1. Do the combinatorial identities of this paper have their analytic counterparts? Our main hope in pursuing the analytic aspects of these results is that we shall find $q$-identities resembling Euler's identities [4, p 277] or Rogers-Ramanujan identities [4, p 290] which will shed light on how to proceed with further study of the identities of this paper.

2 Is it possible to prove the identities of this paper graphically?

3. By using $n$-color partitions we have given an explicit expression for $\sum_{j=1}^{\infty} d(2 j-1)$ Is it possible to find similar expressions for $\sum_{j=1}^{\infty} d(2 j)$ ?

ACKNOWLEDGMENT. The authors would like to thank Dr. Venkatest Raman for his computer assistance

\section{REFERENCES}

[1] AGARWAL, A.K, Rogers-Ramanujam identities for $n$-color partitions, J. Number Theory 28 (1988), 299-305

[2] AGARWAL, A.K. and ANDREWS, G.E, Rogers-Ramanujam identities for partitions with "N copies of N", J. Combin. Theory Ser. A 45, No. 1 (1987), 40-49

[3] AGARWAL, A.K. and MULLEN, G.L., Partitions with "d(a) copies of a", J. Combin. Theory Ser. $A, 48$ (1) (1988), 120-135.

[4] HARDY, G.H and WRIGHT, E M., An Introduction to the Theory of Numbers, Oxford Univ. Press, 5th ed., London, 1979. 


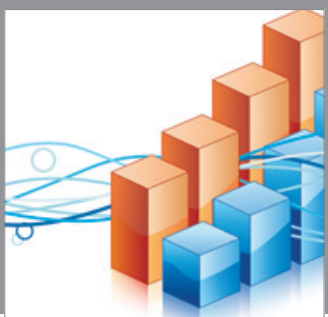

Advances in

Operations Research

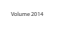

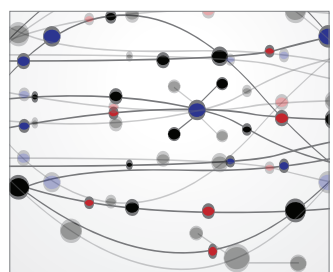

\section{The Scientific} World Journal
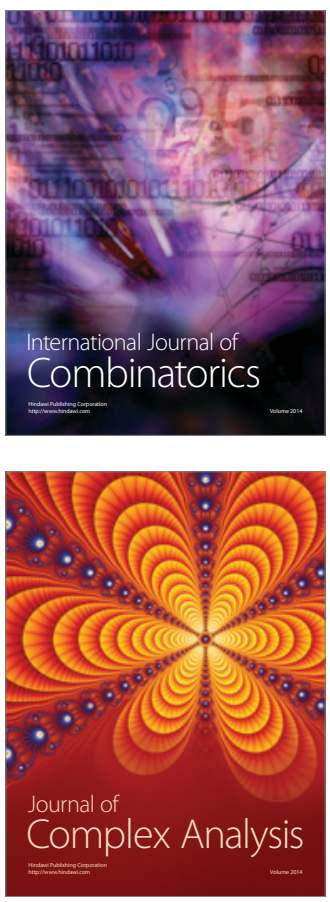

International Journal of

Mathematics and

Mathematical

Sciences
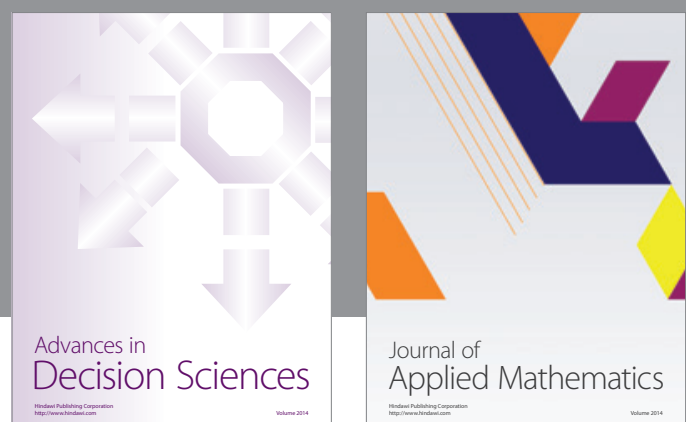

Journal of

Applied Mathematics
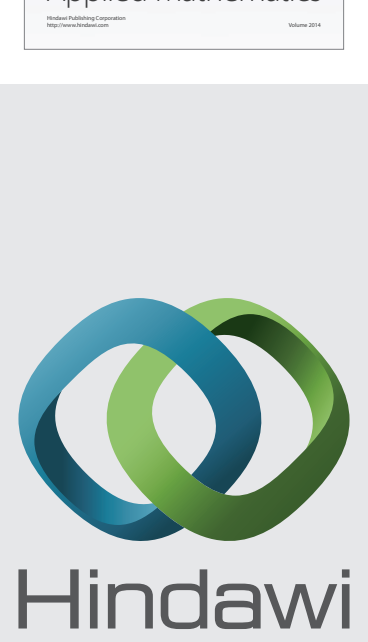

Submit your manuscripts at http://www.hindawi.com
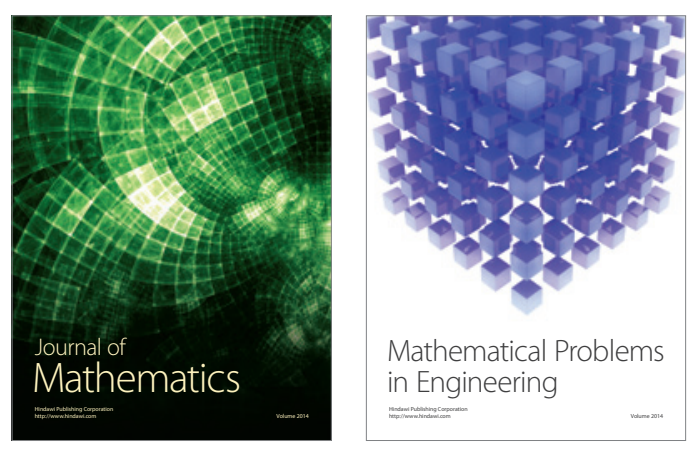

Mathematical Problems in Engineering
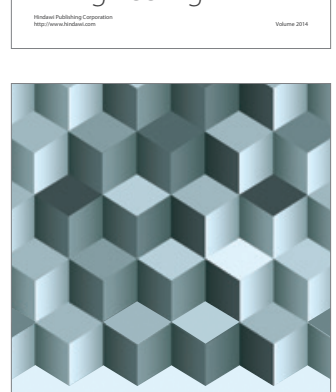

Journal of

Function Spaces
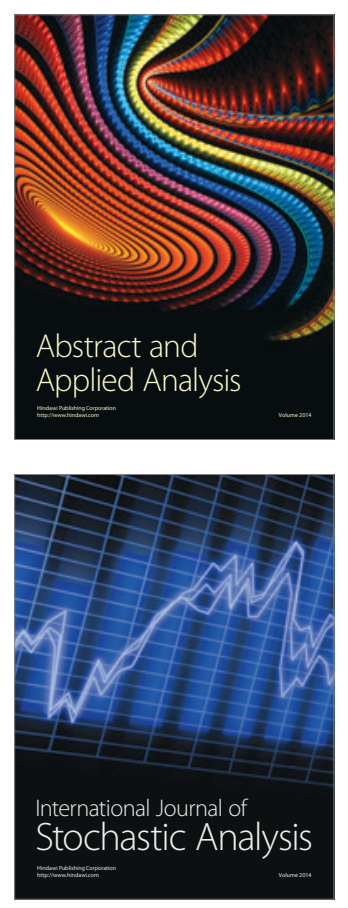

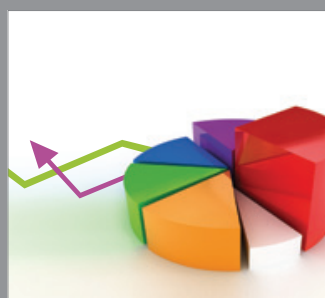

ournal of

Probability and Statistics

Promensencen
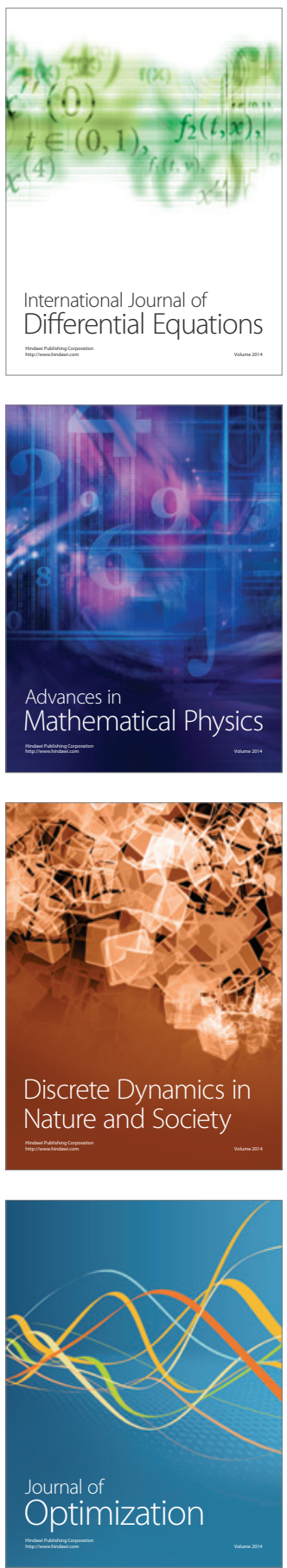